\title{
New approach to measure preference stability
}

\author{
T. González-Artega \\ BORDA and PRESAD Research Groups \\ Multidisciplinary Institute of Enterprise (IME), \\ University of Valladolid, \\ E47011 Valladolid, Spain \\ Email: teresag@eio.uva.es
}

\author{
R. de Andrés Calle \\ BORDA Research Unit, PRESAD Research Group \\ Multidisciplinary Institute of Enterprise (IME), \\ University of Salamanca, \\ E37007 Salamanca, Spain \\ Email:rocioac@usal.es
}

\begin{abstract}
A non-traditional approach about the measurement of agents' preference stability is introduced. This contribution focus on measuring preference consensus at different moments under the assumption of considering the following evaluations: approved, undecided and disapproved. To this aim, the concept of preference stability measure is defined as well as a particular one, the sequential preference stability measure, taking into account any two successive time moments. Finally and in order to highlight the good behaviour of novel measures, some properties are also provided.
\end{abstract}

\section{INTRODUCTION}

Several research fields such as Economics, Social Choice, Marketing, Decision Analysis have been paying attention to intertemporal decision making problems.

In the traditional theory literature, preferences have mainly been considered constant along time [1], but some of current studies are focused on checking if preferences are constant over time [2]. From an empirical point of view, preference stability has been studied using small samples in short time periods considering the following type of preferences, the risk preferences [3], [4]. In recent years, there has been an increasing interest in works about time preference [5], [6], [7], but only a few contributions study the stability of social preferences [8].

From another point of view, there has been an increase in the number of studies that considers changes in preferences as consequence of shocks such as illness, civil wars, natural disasters, etc. [9], [10], [11], [12]. In addition, other research areas like Game Theory have been dealing with the aforementioned problem [13], [14], [15].

Taking into account the previous literature on measurement of preference stability, this contribution addresses an intertemporal decision making problem where agents or experts express their opinions on an alternative/candidate/option over different time moments. Particularly, agents express their opinions on the alternative under study at different times showing their approving, indecision or disapproving on it.

Under the assumption of this framework, the objective of this contribution is to determine how much stability agents' opinions conveys to the group on the alternative along time. For this propose, a new approach to measure preference stability from a non-traditional perspective is defined, the preference stability measure. This measurement takes values in the unit interval considering value 1 full stability and value 0 total lack of stability. Moreover, an specific formulation of the preference stability measure is introduced, the sequential preference stability measure as well as a study of its analytic properties. Under this approach, the stability of preferences is understood like the probability that for a randomly chosen moment of time, two randomly chosen agents have the same opinion at such a time and its consecutive.

The paper is structured as follows. Section 2 establishes the notation necessary to be the contribution self-contained. Section 3 introduces our proposal to measure preference stability: the preference stability measure. Moreover, an specific type of this measure, the sequential preference stability measure, is presented as well as its properties. Finally, some concluding remarks are provided.

\section{NOTATION}

Let $\mathbf{N}=\{1,2, \ldots, N\}$ a set of agents or experts. Agents express their opinions on an alternative, $x$, at different time moments $\mathbf{T}=\left\{t_{1}, \ldots, t_{T}\right\}$.

From now on, the notation used to formalize theses assessments is the following:

Definition 1: A temporal preference profile of a set of agents $\mathbf{N}$ on an alternative $x$ at $T$ different time moments is an $N \times T$ matrix

$$
\mathbf{P}=\left(\begin{array}{ccc}
P_{1 t_{1}} & \ldots & P_{1 t_{T}} \\
\vdots & \ddots & \vdots \\
P_{N t_{1}} & \ldots & P_{N t_{T}}
\end{array}\right)_{N \times T}
$$

where $P_{i t_{j}}$ is the opinion of the agent $i$ over alternative $x$ at $t_{j}$ moment, in the sense

$P_{i t_{j}}= \begin{cases}1 & \text { if agent } i \text { approves } x \text { at the } t_{j} \text { time, } \\ 0.5 & \text { if agent } i \text { is undecided on } x \text { at the } t_{j} \text { time, } \\ 0 & \text { otherwise. }\end{cases}$

Let $\mathbb{P}_{N \times T}$ denote the set of all such $N \times T$ matrices. For simplicity of notation, $(1)_{N \times T}$ is the $N \times T$ matrix whose cells are universally equal to $1,(0.5)_{N \times T}$ is the $N \times T$ 
matrix whose cells are universally equal to 0.5 and $(0)_{N \times T}$ is the $N \times T$ matrix whose cells are universally equal to 0 .

A temporal preference profile $\mathbf{P}$ is unanimous if alternative $x$ is approved (resp. undecided or disapproved) over $\mathbf{T}$ by all agents. In matrix terms, if the time preference profile $\mathbf{P} \in \mathbb{P}_{N \times T}$ is constant, $\mathbf{P}=(1)_{N \times T}$ (resp. $\mathbf{P}=(0.5)_{N \times T}$ or $\left.\mathbf{P}=(0)_{N \times T}\right)$.

Any permutation $\sigma$ of the agents $\{1,2, \ldots, N\}$ determines a temporal preference profile $\mathbf{P}^{\sigma}$ by permutation of the rows of $\mathbf{P}$, that is, row $i$ of the profile $\mathbf{P}^{\sigma}$ is row $\sigma(i)$ of the profile $\mathbf{P}$.

For each temporal preference profile $\mathbf{P}, \mathbf{P}_{S}$ is the restriction to a subset of agents, an agent-subprofile on the agents in $S \subseteq \mathbf{N}$, and it emerges from selecting the rows of $\mathbf{P}$ that are associated with the respective agents in $S$.

For each temporal preference profile $\mathbf{P}, \mathbf{P}^{I}$ is the restriction to a subset of consecutive moments of time, temporalsubprofile on the moments of time in $I \subseteq \mathbf{T}$, and it emerges from selecting consecutive columns of $\mathbf{P}$ that are associated with the respective moments of time in $I$. Any partition $\left\{I_{1}, \ldots, I_{p}\right\}$ of $\mathbf{P}$ generates a decomposition of $\mathbf{P}$ into temporal-subprofiles $\mathbf{P}^{I_{1}}, \ldots, \mathbf{P}^{I_{p}}$ where $\mathbf{P}^{I_{1}} \cup \ldots \cup \mathbf{P}^{I_{p}}=\mathbf{P}$.

An extension of a temporal preference profile $\mathbf{P}$ of a group of agents $\mathbf{N}$ at $\mathbf{T}=\left\{t_{1}, \ldots, t_{T}\right\}$ is a temporal preference profile $\overline{\mathbf{P}}$ at $\overline{\mathbf{T}}=\left\{t_{1}, \ldots, t_{T}, t_{T+1}, \ldots, t_{T+q}\right\}$ such that the restriction of $\overline{\mathbf{P}}$ to the first $T$ moments of time of $\overline{\mathbf{T}}$ coincides with $\mathbf{P}$.

A replication of a temporal preference profile $\mathbf{P}$ of a group of agents $\mathbf{N}$ on alternative $x$ is the temporal preference profile $\mathbf{P} \uplus \mathbf{P} \in \mathbb{P}_{2 N \times T}$ obtained by duplicating each row of $\mathbf{P}$, in the sense that rows $r$ and $N+r$ of $\mathbf{P} \uplus \mathbf{P}$ are row $r$ of $\mathbf{P}$, for each $r=1, \ldots, N$.

For each temporal preference profile $\mathbf{P}$ on alternative $x$, $n_{0}^{t_{j}}$ denotes the number of agents that disapprove $x$ at the $t_{j}$ moment of time, $n_{0.5}^{t_{j}}$ denotes the number of agents that are undecided on $x$ at $t_{j}$, and $n_{1}^{t_{j}}$ denotes the number of agents that approve alternative $x$ at the $t_{j}$ moment of time. Therefore, $N=n_{0}^{t_{j}}+n_{0.5}^{t_{j}}+n_{1}^{t_{j}}$ for each $t_{j} \in \mathbf{T}$. See Table I for enhancing the understanding.

In addition, $n_{0,0}^{t_{j}, t_{j+1}}$ denotes the number of agents that disapprove alternative $x$ at $t_{j}$ and keep their opinion at the following point of time $t_{j+1}$. Analogously, $n_{0.5,0.5}^{t_{j}, t_{j+1}}$ denotes the number of agents that are undecided on alternative $x$ at $t_{j}$ and $t_{j+1}$. In the same vein, $n_{1,1}^{t_{j}, t_{j+1}}$ denotes the number of agents that approve alternative $x$ at $t_{j}$ and keep their opinion at the following point of time $t_{j+1}$.

In this regard, $n_{0,1}^{t_{j}, t_{j+1}}$ is the number of agents that disapprove alternative $x$ at $t_{j}$ but change their opinion at $t_{j+1}$, and $n_{1,0}^{t_{j}, t_{j+1}}$ is the number of agents that approve alternative $k$ at $t_{j}$ but change their opinion at $t_{j+1} \cdot n_{0.5,1}^{t_{j}, t_{j+1}}$ and $n_{0.5,0}^{t_{j}, t_{j+1}}$ denote the number of agents that are undecided at $t_{j}$

\begin{tabular}{|c|c|c|c|c|}
\hline$\underbrace{}_{t_{j}}{ }^{t_{j+1}}$ & No & Undecided & Yes & \\
\hline No & $n_{0,0}^{t_{j}, t_{j+1}}$ & $n_{0,0.5}^{t_{j}, t_{j+1}}$ & $n_{0,1}^{t_{j}, t_{j+1}}$ & $n_{0}^{t_{j}}$ \\
\hline Undecided & $n_{0.5,0}^{t_{j}, t_{j+1}}$ & $\begin{array}{c}n_{0.5,0.5}^{t_{j}, t_{j+1}} \\
\end{array}$ & $n_{0.5,1}^{t_{j}, t_{j+1}}$ & $n_{0.5}^{t_{j}}$ \\
\hline \multirow[t]{2}{*}{ Yes } & $n_{1,0}^{t_{j}, t_{j+1}}$ & $n_{1,0.5}^{t_{j}, t_{j+1}}$ & $n_{1,1}^{t_{j}, t_{j+1}}$ & $n_{1}^{t_{j}}$ \\
\hline & $n_{0}^{t_{j+1}}$ & $n_{0.5}^{t_{j+1}}$ & $n_{1}^{t_{j+1}}$ & $N$ \\
\hline
\end{tabular}

Table I: Condensed table of notation

but change their opinion at $t_{j+1}$ for approving or disapproving $x$, respectively. Similarly, $n_{0,0.5}^{t_{j}, t_{j+1}}$ and $n_{1,0.5}^{t_{j}, t_{j+1}}$ denote the number of agents that disapprove and approve $x$ at $t_{j}$, respectively, but change their opinion at $t_{j+1}$ for undecided. For each $t_{j} \in \mathbf{T}, n_{0}^{t_{j}}=n_{0,0}^{t_{j}, t_{j+1}}+n_{0,0.5}^{t_{j}, t_{j+1}}+n_{0,1}^{t_{j}, t_{j+1}}$, $n_{0.5}^{t_{j}}=n_{0.5,0}^{t_{j}, t_{j+1}}+n_{0.5,0.5}^{t_{j}, t_{j+1}}+n_{0.5,1}^{t_{j}, t_{j+1}}$ and likewise $n_{1}^{t_{j}}=n_{1,1}^{t_{j}, t_{j+1}}+n_{1,0.5}^{t_{j}, t_{j+1}}+n_{1,0}^{t_{j}, t_{j+1}}$.

For the purpose of clarifying the use of the previous notation, the following illustrative example is introduced.

Example 1: Let $\mathbf{N}=\{1,2, \ldots, 12\}$ be a set of twelve agents that express their opinions on alternative $x$ along four consecutive moments of time $\mathbf{T}=\left\{t_{1}, t_{2}, t_{3}, t_{4}\right\}$. Their temporal preference profile is:

$\mathbf{P}=\left(\begin{array}{ccc}P_{1 t_{1}} & \ldots & P_{1 t_{4}} \\ \vdots & \ddots & \vdots \\ P_{12 t_{1}} & \ldots & P_{12 t_{4}}\end{array}\right)=\left(\begin{array}{cccc}0 & 0 & 0.5 & 0.0 \\ 0.5 & 0 & 1.0 & 0.5 \\ 1 & 0.0 & 1 & 0 \\ 0 & 1 & 0.5 & 0 \\ 0 & 0 & 0 & 1 \\ 1 & 0 & 0 & 0 \\ 0.5 & 1 & 0 & 0 \\ 0.5 & 1 & 0 & 1 \\ 0.5 & 0.5 & 1 & 0.5 \\ 1 & 1 & 1 & 0.5 \\ 0.5 & 0.5 & 0.5 & 1 \\ 0.5 & 0 & 0 & 0\end{array}\right)_{12 \times 4}$

This temporal preference profile can be summarized in a table containing the number of agents who approve, are undecided or disapprove alternative $x$ at each moment of time $t_{j}$ as well as the number of agents that keep or change their opinion during consecutive time moments (see Table II).

\section{THE PREFERENCE STABILITY MEASURE: Definition AND PROPERTIES}

In this section, our proposal of preference stability measure as well as its properties are introduced. Concretely, the notion of preference stability is considered in the same vein that the notion of Bosch's consensus [16]. This seems natural because the measurement of preference stability resembles the notion 


\begin{tabular}{l|lll|l}
\hline$t_{1}$ & No & Undecided & Yes & \\
\hline No & $n_{0,0}^{t_{1}, t_{2}}=2$ & $n_{0,0.5}^{t_{1}, t_{2}}=0$ & $n_{0,1}^{t_{1}, t_{2}}=1$ & $n_{0}^{t_{1}}=3$ \\
Undecided & $n_{0.5,0}^{t_{1}, t_{2}}=2$ & $n_{0.5,0.5}^{t_{1}, t_{2}}=2$ & $n_{0.5,1}^{t_{1}, t_{2}}=2$ & $n_{0.5}^{t_{1}}=6$ \\
Yes & $n_{1,0}^{t_{1}, t_{2}}=2$ & $n_{1,0.5}^{t_{1}, t_{2}}=0$ & $n_{1,1}^{t_{1}, t_{2}}=1$ & $n_{1}^{t_{1}}=3$ \\
\hline & $n_{0}^{t_{2}}=6$ & $n_{0.5}^{t_{2}}=2$ & $n_{1}^{t_{2}}=4$ & $N=12$
\end{tabular}

\begin{tabular}{l|lll|l}
\hline$t_{2}$ & No & Undecided & Yes & \\
\hline No & $n_{0,0}^{t_{2}, t_{3}}=3$ & $n_{0,0.5}^{t_{2}, t_{3}}=1$ & $n_{0,1}^{t_{2}, t_{3}}=2$ & $n_{0}^{t_{2}}=6$ \\
Undecided & $n_{0.5,0}^{t_{2}, t_{3}}=0$ & $n_{0.5,0.5}^{t_{2}, t_{3}}=1$ & $n_{0.5,1}^{t_{2}, t_{3}}=1$ & $n_{0.5}^{t_{2}}=2$ \\
Yes & $n_{1,0}^{t_{2}, t_{3}}=2$ & $n_{1,0.5}^{t_{2}, t_{3}}=1$ & $n_{1,1}^{t_{2}, t_{3}}=1$ & $n_{1}^{t_{2}}=4$ \\
\hline & $n_{0}^{t_{3}}=5$ & $n_{0.5}^{t_{3}}=3$ & $n_{1}^{t_{3}}=4$ & $N=12$
\end{tabular}

\begin{tabular}{l|lll|l} 
& \multicolumn{3}{|c}{} & \\
$t_{3} t_{4}$ & No & Undecided & Yes & \\
\hline No & $n_{0,0}^{t_{3}, t_{4}}=3$ & $n_{0,0.5}^{t_{3}, t_{4}}=0$ & $n_{0,1}^{t_{3}, t_{4}}=2$ & $n_{0}^{t_{3}}=5$ \\
Undecided & $n_{0.5,0}^{t_{3}, t_{4}}=2$ & $n_{0.5,0.5}^{t_{3}, t_{4}}=0$ & $n_{0.5,1}^{t_{3}, t_{4}}=1$ & $n_{0.5}^{t_{3}}=3$ \\
Yes & $n_{1,0}^{t_{3}, t_{4}}=1$ & $n_{1,0.5}^{t_{3}, t_{4}}=3$ & $n_{1,1}^{t_{3}, t_{4}}=0$ & $n_{1}^{t_{3}}=4$ \\
\hline & $n_{0}^{t_{4}}=6$ & $n_{0.5}^{t_{4}}=3$ & $n_{1}^{t_{4}}=3$ & $N=12$
\end{tabular}

Table II: Condensed table of notation for Example 1

of measurement of consensus over time, in the sense that the maximum value captures the notion of full stability, that is unanimity along time, while the minimum value captures the notion of total lack of stability, that is, total disagreement along time.

From the Social Choice literature, it is possible to point out the consensus measurement proposed by Alcalde-Unzu and Vorsatz [17], Alcantud et al. [18], Alcantud, de Andrés Calle and Cascón [19], García-Lapresta and Pérez-Román [20] and González-Arteaga et al. [21]. Additionally, there are several studies related to consensus problem from the Decision Making Theory like the approaches proposed by González-Arteaga et al. [22], González-Pachón and Romero [23], González-Pachón et al. [24], Herrera-Viedma et al. [25], and so on.

Taking into account the aforementioned arguments, our novel approach to measure preference stability is now presented.

Definition 2: A preference stability measure for a group of agents $\mathbf{N}=\{1, \ldots, N\}$ on an alternative $x$ is a mapping

$$
\psi: \mathbb{P}_{N \times T} \rightarrow[0,1]
$$

that assigns a number $\psi(\mathbf{P}) \in[0,1]$ to each temporal preference profile $\mathbf{P}$, with the properties:

i) $\psi(\mathbf{P})=1$ if and only if $\mathbf{P}$ is unanimous (full stability).

ii) $\psi\left(\mathbf{P}^{\sigma}\right)=\psi(\mathbf{P})$ for each permutation $\sigma$ of the agents and $\mathbf{P} \in \mathbb{P}_{N \times T}$ (anonymity).
A preference stability measure is a collection of preference stability measures for each group of agents $\mathbf{N}$.

Our proposal unlike Bosch's contribution does not require neutrality property, time moments can be exchanged, due to the fact that time order is an essential aspect to measure the stability of preferences.

Now a particular preference stability measure is introduced. Formally:

Definition 3: The sequential preference stability measure for a group of agents $\mathbf{N}=\{1, \ldots, N\}$ on an alternative $x$ is the mapping $\psi_{S}: \mathbb{P}_{N \times T} \rightarrow[0,1]$ given by

$$
\begin{aligned}
& \psi_{S}(\mathbf{P})= \\
&=\frac{1}{T-1} \cdot \frac{\sum_{j=1}^{j=T-1} n_{0,0}^{t_{j}, t_{j+1}} \cdot\left(n_{0,0}^{t_{j}, t_{j+1}}-1\right)}{N(N-1)} \\
&+\frac{1}{T-1} \cdot \frac{\sum_{j=1}^{j=T-1} n_{0.5,0.5}^{t_{j}, t_{j+1}} \cdot\left(n_{0.5,0.5}^{t_{j}, t_{j+1}}-1\right)}{N(N-1)} \\
&+\frac{1}{T-1} \cdot \frac{\sum_{j=1}^{j=T-1} n_{1,1}^{t_{j}, t_{j+1}} \cdot\left(n_{1,1}^{t_{j}, t_{j+1}}-1\right)}{N(N-1)}
\end{aligned}
$$

Intuitively, it measures the probability that for a randomly chosen moment of time, two randomly chosen agents of a group have the same opinion upon an alternative at the moment of time selected and its consecutive.

It is easy to check that Definition 3 provides a preference stability measure.

Example 2: For the temporal preference profile in Example 1, the computations obtained are the following:

$$
\begin{aligned}
\psi_{S}(\mathbf{P}) & = \\
& =\frac{1}{3} \cdot \frac{2(2-1)+3(3-1)+3(3-1)}{12(11)}+ \\
& +\frac{1}{3} \cdot \frac{2(2-1)+1(1-1)+0(0-1)}{12(11)}+ \\
& +\frac{1}{3} \cdot \frac{1(1-1)+1(1-1)+0(0-1)}{12(11)}= \\
& =0.04
\end{aligned}
$$

In this case, the sequential stability measure takes a value near zero because the opinions of two agents hardly ever coincidence in two successive time moments. 
Some desirable properties of the sequential preference stability measure are defined bellow.

\section{Properties $^{1}$ :}

1) Reversal invariance among decided agents: This property shows that the main aspect of the sequential preference stability measure is the stability of agents' opinions more than an specific value. If the 0's are changed for 1 's and vice verse (undecided agents do not change), then the sequential preference stability measure reminds equal. Formally:

Let $\mathbf{P}^{c}$ be the complementary temporal preference profile of $\mathbf{P}$ defined by $\mathbf{P}^{c}=(1)_{N \times T}-\mathbf{P}$. If $\psi_{S}$ verifies reversal invariance among decided agents then $\psi_{S}\left(\mathbf{P}^{c}\right)=\psi_{S}(\mathbf{P})$.

2) Temporal reducibility: It means that the stability of a temporal preference profile is the average of the sequential preference stability measures of all its consecutive temporal-subprofiles of two consecutive moments of time. Formally:

Let $\mathbf{P} \in \mathbb{P}_{N \times T}$ be a temporal preference profile. We say that $\psi_{S}$ verifies time-reducibility if

$$
\psi_{S}(\mathbf{P})=\frac{1}{T-1} \sum_{j=1}^{T-1} \psi_{S}\left(\mathbf{P}^{I_{j, j+1}}\right)
$$

where $\mathbf{P}^{I_{j, j+1}} \in \mathbb{P}_{N \times 2}$ is the temporal-subprofile of $\mathbf{P}$ containing the columns corresponding to times $t_{j}$ and $t_{j+1}$.

3) Convexity: It means the sequential preference stability measure of a temporal preference profile is a weighted average of the measures of any decomposition of $\mathbf{P}$ into consecutive temporal-subprofiles. Formally:

For each temporal preference profile $\mathbf{P} \in \mathbb{P}_{N \times T}$, and each decomposition of $\mathbf{P}$ into two consecutive temporalsubprofiles, $\mathbf{P}^{I_{1}} \in \mathbb{P}_{N \times\left(k_{1}+1\right)}$ and $\mathbf{P}^{I_{2}} \in \mathbb{P}_{N \times\left(T-k_{1}\right)}$ with $I_{1}=\left\{t_{1}, \ldots, t_{k_{1}+1}\right\}$ and $I_{2}=\left\{t_{k_{1}+1}, \ldots, t_{T}\right\}$, and $\left(\left|I_{1}\right|-1\right)+\left(\left|I_{2}\right|-1\right)=T-1$

$\psi_{S}(\mathbf{P})=\frac{\left(\left|I_{1}\right|-1\right) \cdot \psi_{S}\left(\mathbf{P}^{I_{1}}\right)+\left(\left|I_{2}\right|-1\right) \cdot \psi_{S}\left(\mathbf{P}^{I_{2}}\right)}{T-1}$

4) Replication monotonicity: When a non-unanimous temporal preference profile is replicated, its sequential preference stability measure increases. Formally:

Let $\mathbf{P} \in \mathbb{P}_{N \times T}$ be a non unanimous temporal preference profile then

$$
\psi_{S}(\mathbf{P} \uplus \mathbf{P})>\psi_{S}(\mathbf{P})
$$

\footnotetext{
${ }^{1}$ The proofs of the properties are not included in this contribution because the limited space, but they can be provided if they were required.
}

In addition, for an unanimous time preference profile $\mathbf{P} \in \mathbb{P}_{N \times T}$, by Definition 3, $\psi_{S}$ verifies

$$
\psi_{S}(\mathbf{P} \uplus \mathbf{P})=\psi_{S}(\mathbf{P})=1
$$

5) Minimum stability: If all agents express their opinions at $t_{j}$ and change their opinions at $t_{j+1}$, then the sequential preference stability measure takes a zero value. It also happens when there are at most two agents keeping their opinion at $t_{j}$ and $t_{j+1}$, but their opinions do not coincide each other. Formally:

Let $\mathbf{P} \in \mathbb{P}_{N \times T}$ be a temporal preference profile such that there is at most one agent who has the same opinion at $t_{j}$ and $t_{j+1}$ for $j \in\{1, \ldots T\}$, that is, $n_{0,0}^{t_{j}, t_{j+1}} \leq 1$, $n_{0.5,0.5}^{t_{j}, t_{j+1}} \leq 1$ and $n_{1,1}^{t_{j}, t_{j+1}} \leq 1$ for all $j \in \mathbf{T}$. Then, $\psi_{S}(\mathbf{P})=0$.

6) Breaking minimum stability: In order to break the minimum stability it is needed that at least the opinions of two agents coincide at the same moment of time and the next one. Formally:

Let $\mathbf{P} \in \mathbb{P}_{N \times T}$ be a temporal preference profile such that there exists at least a $k, k \in \mathbf{T}$, such that $n_{0,0}^{t_{k}, t_{k+1}}>1$ or $n_{0.5,0.5}^{t_{k}, t_{k+1}}>1$ or $n_{1,1}^{t_{k}, t_{k+1}}>1$, then $\psi_{S}(\mathbf{P})>0$.

7) Temporal monotonicity: Consider two temporal preference profiles, $\mathbf{P}$ and $\mathbf{P}^{\prime}$, that coincide in all their elements excepting the opinion of an agent $m \in \mathbf{N}$, at $t_{k}$ and $t_{k+1}$. Concretely, this agent has different opinion at $t_{k}$ and $t_{k+1}$ in $\mathbf{P}: P_{m t_{j}} \neq P_{m t_{j+1}}$, and the agent's opinion is the same at $t_{k}$ and $t_{k+1}$ in $\mathbf{P}^{\prime}$ : $P_{m t_{j}}^{\prime}=P_{m t_{j+1}}^{\prime}$. In this case, the sequential preference stability measure verifies $\psi_{S}\left(\mathbf{P}^{\prime}\right) \geq \psi_{S}(\mathbf{P})$. Formally:

Let $\mathbf{P}, \mathbf{P}^{\prime} \in \mathbb{P}_{N \times T}$ be temporal preference profiles such that:

a) $P_{i t_{j}}=P_{i t_{j}}^{\prime}, i \in\{\mathbf{N} \backslash\{m\}\}, t_{j} \in\left\{\mathbf{T} \backslash\left\{t_{k}, t_{k+1}\right\}\right\}$,

b) $P_{m t_{k}} \neq P_{m t_{k+1}}, m \in \mathbf{N}, t_{k}, t_{k+1} \in \mathbf{T}$,

c) $P_{m t_{k}}^{\prime}=P_{m t_{k+1}}^{\prime}, m \in \mathbf{N}, t_{k}, t_{k+1} \in \mathbf{T}$.

Then, $\psi_{S}\left(\mathbf{P}^{\prime}\right) \geq \psi_{S}(\mathbf{P})$.

8) Convergence to full stability: If new moments of time are repeatedly introduced into the problem and all agents have the same opinion at them, then the sequential preference stability measure approaches 1 . Formally:

Suppose that $q$ moments of time $t_{T+1}, \ldots t_{T+q}$ are added to $\mathbf{T}$, and at these new moments of time the alternative $x$ is unanimously approved (resp. unanimously 
undecided or unanimously disapproved) by all agents. If the introduction of new moments of time does not affect agents' opinions in past times, then the sequential time cohesiveness measure of the extended temporal preference profile $\overline{\mathbf{P}}^{(q)} \in \mathbb{P}_{N \times(T+q)}$ approaches 1 when $q$ tends to infinity.

$$
\lim _{q \rightarrow \infty} \psi_{S}\left(\overline{\mathbf{P}}^{(\mathbf{q})}\right)=1
$$

\section{CONCLUDING REMARKS}

Research in the subject of preference stability has made progress mostly in Economics. The aim of this paper is to manage the problem of measuring preference stability from a non-traditional perspective. In order to set forth the context of our research a framework is establihsed where agents express their opinions on an alternative at different moments considering the following evaluations: approved, undecided and disapproved. The general notion of preference stability measure is introduced. Then, a specific formulation is developed with particular regard to any two successive time moments. In this way, the sequential preference stability measure is proposed. Moreover, some meaningful properties which make our proposal compelling are also provided.

Overall, the proposals of this contribution have a range of implications for future research. Many problems on preference stability from a diversity of fields can be faced by our approach such as the consumers' preferences, risk preference, and so on.

\section{ACKNOWLEDGMENT}

The authors acknowledge financial support by the Spanish Ministerio de Ciencia e Innovación under Project Project ECO2016-77900-P.

\section{REFERENCES}

[1] G. Loewenstein and E. Angner, "Predicting and indulging changing preferences," Time and decision: Economic and psychological perspectives on intertemporal choice, pp. 351-391, 2003.

[2] Y. Chuang and L. Schechter, "Stability of experimental and survey measures of risk, time, and social preferences: A review and some new results," Journal of Development Economics, vol. 117, pp. 151 - 170, 2015.

[3] C. Dave, C. Eckel, C. Johnson, and C. Rojas, "Eliciting risk preferences: When is simple better?" Journal of Risk and Uncertainty, vol. 41, no. 3, pp. 219-243, 2010.

[4] C. Sahm, "How much does risk tolerance change?" Quarterly Journal of Finance, vol. 02, no. 04, 2012.

[5] J. Doyle, "Survey of time preference, delay discounting models," Judgment and Decision Making, vol. 8, no. 2, pp. 116-135, 2013.

[6] E. Krupka and M. Stephens, "The stability of measured time preferences," Journal of Economic Behavior and Organization, vol. 85, no. 1, pp. 11-19, 2013.

[7] S. Meier and C. Sprenger, "Temporal stability of time preferences," Review of Economics and Statistics, vol. 97, no. 2, pp. 273-286, 2015.

[8] F. Carlsson, O. Johansson-Stenman, and P. Nam, "Social preferences are stable over long periods of time," Journal of Public Economics, vol. 117, pp. 104-114, 2014.

[9] L. Cameron and M. Shah, "Risk-taking behavior in the wake of natural disasters," Journal of Human Resources, vol. 50, no. 2, pp. 484-515, 2015.
[10] P. Gerrans, R. Faff, and N. Hartnett, "Individual financial risk tolerance and the global financial crisis," Accounting and Finance, vol. 55, no. 1, pp. 165-185, 2015.

[11] P. Jakiela, E. Miguel, and V. te Velde, "You've earned it: estimating the impact of human capital on social preferences," Experimental Economics, vol. 18, no. 3, pp. 385-407, 2015.

[12] S. Necker and M. Ziegelmeyer, "Household risk taking after the financial crisis," The Quarterly Review of Economics and Finance, vol. 59, pp. $141-160,2016$

[13] L. Anderson and J. Mellor, "Are risk preferences stable? Comparing an experimental measure with a validated survey-based measure," Journal of Risk and Uncertainty, vol. 39, no. 2, pp. 137-160, 2009.

[14] J. Brandts and G. Charness, "Hot vs. cold: Sequential responses and preference stability in experimental games," Experimental Economics, vol. 2 , no. 3, pp. 227-238, 2000.

[15] A. Reynaud and S. Couture, "Stability of risk preference measures: Results from a field experiment on french farmers," Theory and Decision, vol. 73, no. 2, pp. 203-221, 2012.

[16] R. Bosch, "Characterizations of voting rules and consensus measures," Ph.D. dissertation, Tilburg University, 2005.

[17] J. Alcalde-Unzu and M. Vorsatz, "Do we agree? Measuring the cohesiveness of preferences," Theory and Decision, vol. 80, pp. 313-339, 2016.

[18] J. Alcantud, R. de Andrés Calle, and J. Cascón, "On measures of cohesiveness under dichotomous opinions: Some characterizations of approval consensus measures," Information Sciences, vol. 240, pp. 45$55,2013$.

[19] _ "Pairwise dichotomous cohesiveness measures," Group Decision and Negotiation, vol. 24, no. 5, pp. 833-854, 2015.

[20] J. L. García-Lapresta and D. Pérez-Román, "Measuring consensus in weak orders," in Consensual Processes, ser. Studies in Fuzziness and Soft Computing, E. Herrera-Viedma, J. L. García-Lapresta, J. Kacprzyk, M. Fedrizzi, H. Nurmi, and S. Zadrozny, Eds. Springer Berlin Heidelberg, 2011, vol. 267, pp. 213-234.

[21] T. González-Arteaga, J. Alcantud, and R. de Andrés Calle, "A cardinal dissensus measure based on the Mahalanobis distance," European Journal of Operational Research, vol. 251, no. 2, pp. 575 - 585, 2016.

[22] T. González-Arteaga, R. de Andrés Calle, and F. Chiclana, "A new measure of consensus with reciprocal preference relations: The correlation consensus degree," Knowledge-Based Systems, vol. 107, pp. 104-116, 2016.

[23] J. González-Pachón and C. Romero, "Aggregation of ordinal and cardinal preferences: a framework based on distance functions," Journal of Multi-Criteria Decision Analysis, vol. 15, no. 3-4, pp. 79-85, 2009.

[24] J. González-Pachón, C. Romero, and L. Díaz-Balteiro, "How to combine inconsistent ordinal and cardinal preferences: a satisficing modelling approach," Computers and Industrial Engineering, vol. 67, pp. 168-172, 2014.

[25] E. Herrera-Viedma, F. Herrera, and F. Chiclana, "A consensus model for multiperson decision making with different preference structures," IEEE Transactions on Systems, Man, and Cybernetics - Part A: Systems and Humans, vol. 32, no. 3, pp. 394-402, 2002. 